\title{
CONTEÚDO DE CARBONO ORGÂNICO EM PLANOSSOLO HÁPLICO SOB SISTEMAS DE MANEJO DO ARROZ IRRIGADO $^{(1)}$
}

\author{
Carla Machado da Rosa ${ }^{(2)}$, Rosa Maria Vargas Castilhos ${ }^{(3)}$, Eloy Antonio \\ Pauletto $^{(3)}$, Clenio Nailto Pillon ${ }^{(4)}$ \& Otávio dos Anjos Leal ${ }^{(5)}$
}

\begin{abstract}
RESUMO
Nos solos de várzea da região Sul do Rio Grande do Sul, onde um milhão de hectares são cultivados com arroz irrigado por alagamento em diferentes sistemas de manejo, a influência da condição de má drenagem e alternância entre ciclos de oxidação e redução sobre o conteúdo de C orgânico do solo (COS) é pouco conhecida. Este estudo foi realizado em um experimento de longa duração (21 anos), localizado no município do Capão do Leão, RS, com o objetivo de avaliar o efeito de sistemas de manejo do arroz irrigado sobre os estoques de COS e das frações físicas da matéria orgânica em um Planossolo Háplico. O fracionamento físico da matéria orgânica do solo foi realizado pelo método densimétrico, em amostras de solo coletadas das camadas de $0-0,025 ; 0,025-0,05 ; 0,05-0,10 ;$ e $0,10-0,20 \mathrm{~m}$, nos tratamentos: ST- Sistema tradicional de cultivo um ano com arroz (preparo convencional) e dois anos com pousio; APC-Sistema de cultivo contínuo de arroz (preparo convencional) e controle de invasoras com herbicida; APD- Sucessão azevém/arroz (plantio direto); e SN-Solo mantido com vegetação natural (campo nativo). $O$ sistema com arroz/azevém em plantio direto APD foi mais eficiente em manter os estoques de COS e da fração leve livre até $0,05 \mathrm{~m}$ de profundidade, em comparação com o ST e APC. A fração leve oclusa não se mostrou sensível aos efeitos dos diferentes sistemas de manejo, sugerindo para o solo de várzea baixa eficiência da proteção física da MOS por oclusão em agregados, o que pode estar relacionado aos efeitos da condição de má drenagem no processo de agregação. A umidade excessiva no inverno e o alagamento durante os cultivos de arroz estariam
\end{abstract}

\footnotetext{
(1) Parte da Tese de Doutorado em Agronomia, área de concentração em Solos, da primeira autora apresentada à Universidade Federal de Pelotas - UFPEL. Recebido para publicação em 29 de julho de 2010 e aprovado em 27 de julho de 2011.

(2) Pós-doutoranda do Departamento de Solos da Faculdade de Agronomia da Universidade Federal do Rio Grande do Sul (UFRGS), Caixa Postal 776, CEP 90001-970 Porto Alegre (RS). E-mail: carlamrosa@yahoo.com.br

(3) Professor Associado do Departamento de Solos da Universidade Federal de Pelotas - UFPEL. Caixa Postal 354, CEP 96010900 Pelotas (RS). E-mail: rosamvc@ufpel.tche.br; pauletto@ufpel.edu.br

(4) Pesquisador da Embrapa Clima Temperado. Caixa Postal 403, CEP 96001-970 Pelotas (RS). E-mail: pillon@cpact.embrapa.br

(5) Mestrando em Agronomia, área de concentração em Solos, UFPEL. E-mail: oleal@ibest.com.br
} 
proporcionando agregação transitória entre cultivos. A quantidade de CO nesse Planossolo, tanto entre os sistemas de cultivo quanto em profundidade, deve-se principalmente à fração pesada, que é a fração mais estável e mais difícil de ser degradada.

Termos de indexação: agregação, matéria orgânica, plantio direto, solo de várzea.

\title{
SUMMARY: CONTENT OF SOIL ORGANIC CARBON IN ALBAQUALF SOIL: INFLUENCE OF IRRIGATED RICE MANAGEMENT
}

\begin{abstract}
On paddy soils in the southern region of the State of Rio Grande do Sul, Brazil, irrigated (flooded) rice is grown on 1'000'000 ha, under different soil management systems. The influence of poor drainage and alternating oxidation and reduction cycles on the content of soil organic carbon is still poorly understood. This long-term study (21 years) was conducted in Capão do Leão, RS, Brazil, to evaluate the effect of irrigated rice management systems on soil organic carbon stocks and physical fractions of the organic matter $(O M)$ of an Albaqualf soil. OM in soil samples from the layers 0-0.025; 0.025-0.05; 0.05-0.10 and 0.10-0.20 $\mathrm{m}$ was physically fractionated by densimetry in the treatments: TC-traditional cultivation system: one year rice (conventional tillage) and two years fallow; RCV-continuous cultivation of rice (conventional tillage) and herbicide weed control; RNT-succession ryegrass / rice in no tillage and SN- native pasture. In the system of rice in no tillage (RNT), after 21 years, the values of total organic carbon and the free light fraction stock were better preserved, to a depth of $0.05 \mathrm{~m}$, compared to the systems with rice under conventional tillage TC and RCV. The occluded light fraction was not sensitive to detect the effects of different tillage systems, indicating soil a low efficiency of physical protection of OM by aggregate occlusion in the lowland soil, which may be related to the poor soil drainage in the aggregation process. Excessive moisture in the winter and flooding of the rice crops may induce a transitory aggregation between crops. The amount of organic carbon in this Albaqualf soil, between cultivation systems as well as in depth, consisted mainly of the more stable heavy fraction that is more resistant to degradation.
\end{abstract}

Index terms: aggregation, flooded soil, no-tillage, organic matter.

\section{INTRODUÇÃO}

No Estado do Rio Grande do Sul, os solos de várzea representam cerca de $20 \%$ da área total, em que desses aproximadamente 35 \% são Planossolos, ocupando uma área de mais de 2,5 milhões de hectares (Pinto et al., 2004). Esses solos apresentam característica bastante peculiar, o hidromorfismo, que é motivado pelo relevo predominantemente plano, associado à presença de um horizonte $\mathrm{B}$ de textura argilosa que impermeabiliza o solo, impedindo a infiltração da água. Tais características tornam esses solos propícios ao cultivo do arroz irrigado, em que o preparo do solo, o método de semeadura e o manejo da água definem os sistemas de manejo adotados, com maior ou menor mobilização do solo.

A submissão, entretanto, desses solos a regimes de drenagem e de alagamento (90-100 dias durante o cultivo com arroz irrigado) ocasiona alterações periódicas no ambiente em função das mudanças na microbiota e nos processos químicos que se alternam entre uma condição aeróbia e outra anaeróbia. A alternância desses ciclos pode resultar numa dinâmica da matéria orgânica (MO), nos solos de várzea, diferenciada daquela nos solos oxidados, tanto em relação aos produtos formados quanto à velocidade de decomposição dela. Além disso, muitos fatores podem alterar o conteúdo de carbono orgânico do solo (COS), principalmente quando se faz a conversão de sistemas naturais para a agricultura, alterando as taxas de adição e degradação da MO. No entanto, existem mecanismos químicos e físicos que podem proteger a MO da ação dos microrganismos decompositores, determinando que moléculas de mesma composição e complexidade sejam diferentemente suscetíveis à decomposição. Esses mecanismos são: recalcitrância, interação química ou coloidal e inacessibilidade ou proteção física (Roscoe et al., 2006).

A matéria orgânica do solo (MOS) é, assim, considerada importante indicador da qualidade do solo e bastante sensível às alterações nos sistemas de manejo. Sistemas conservacionistas sem mobilização do solo ou com revolvimento mínimo, utilizando culturas que permitem maior aporte de matéria seca, tendem a preservar ou, até mesmo, a aumentar o estoque de $\mathrm{C}$, principalmente em solos com baixos 
teores de MO, o que também se espera dos solos de várzea. No entanto, pouco se sabe sobre a intensidade com que diferentes sistemas de manejo (preparo do solo e periodicidade de alagamento), principalmente no cultivo do arroz irrigado, podem influenciar nas frações da MOS e nos seus mecanismos de proteção.

As frações lábeis da MOS têm grande influência na atividade biológica e na química de solos alagados e podem ter impacto sobre as emissões de gases de efeito-estufa (Gaunt et al., 1997; Nascimento et al., 2009). Identificar os mecanismos de estabilização da MOS que atuam com maior eficiência nesses solos é essencial para avaliar o potencial de degradação de cada sistema de manejo adotado. A estabilização da MO, seja pela menor acessibilidade dos microrganismos, "proteção física", ou pela formação de complexos estáveis entre a fração orgânica e a mineral, "proteção química", é fundamental para a preservação dos estoques de COS (Roscoe et al., 2006), assim como tem grande importância para a agregação do solo, seguindo-se a teoria da agregação proposta por Tisdall \& Oades (1982), em que a MO é o principal agente ligante para a formação dos agregados.

Nos solos de várzea cultivados com arroz irrigado, além dos sistemas de preparo, o alagamento do solo desestabiliza a sua estrutura, promovendo agregação transitória entre o período drenado e o alagado. Dessa forma, a proteção física da MOS seria um mecanismo pouco eficiente na sua estabilização, e a fração leve oclusa pouco contribuiria para o acúmulo de $\mathrm{C}$ em solos de várzea cultivados (Nascimento et al., 2009).

Nesse contexto, este estudo teve como objetivo avaliar o efeito de sistemas de manejo do arroz irrigado sobre os estoques de C orgânico e das frações densimétricas da MOS em um Planossolo cultivado há 21 anos, na região Sul do Rio Grande do Sul.

\section{MATERIAL E MÉTODOS}

O estudo foi realizado em um experimento que vinha sendo trabalhado desde 1985, em Planossolo Háplico eutrófico solódico, localizado na Estação Experimental de Terras Baixas da Embrapa Clima Temperado, no município do Capão do Leão, RS, em uma área onde, anteriormente, era campo nativo nunca cultivado, com predomínio de espécies gramíneas. O clima da região é $\mathrm{Cfa}$ (C: clima mesotérmico quente, com média do mês de frio entre 3 e $18{ }^{\circ} \mathrm{C}$; f: precipitação pluvial média mensal não inferior a $60 \mathrm{~mm}$ - sempre úmido; e a: temperatura do mês mais quente superior a $22^{\circ} \mathrm{C}$ ), segundo a classificação de Köppen. O solo da área experimental apresentava, em média, na camada de $0-0,20 \mathrm{~m}$, $149 \mathrm{~g} \mathrm{~kg}^{-1}$ de argila, $389 \mathrm{~g} \mathrm{~kg}^{-1}$ de silte e $463 \mathrm{~g} \mathrm{~kg}^{-1}$ de areia, tendo predomínio dos argilominerais caulinita e mistura de minerais 2:1 na fração argila (Castilhos et al., 2002).
Os tratamentos são constituídos por sistemas de cultivo de arroz irrigado, dispostos em parcelas de $600 \mathrm{~m}^{2}$, em blocos ao acaso $(\mathrm{n}=4)$, e que vinham sendo mantidos e trabalhados sempre da mesma forma desde a sua implantação. Para este estudo, em função da intensidade de preparo do solo e da frequência de alagamento, foram selecionados quatro tratamentos: ST - sistema tradicional de cultivo: um ano de arroz irrigado com preparo convencional do solo, semeadura em solo seco e irrigação por alagamento (90-100 dias), seguida de dois anos de pousio; APC - cultivo contínuo de arroz irrigado por alagamento (90-100 dias), com preparo convencional do solo e pousio no inverno; APD - sucessão azevém/arroz irrigado, sob plantio direto (ambas as culturas semeadas diretamente sobre a palha da cultura anterior, sem nenhum revolvimento do solo); e SN - parcela mantida com vegetação natural (campo nativo). As principais espécies que compõem o campo nativo são: Paspalum notatum, Paspalum pumilum, Paspalum modestum, Paspalum dilatatum, Desmodium incanum, Andropogon lateralis, Ischaemum minus, Leersia Lexandra e Axonopus offinis.

As amostras de solo em cada parcela foram coletadas em outubro de 2006, em trincheiras, nas camadas de $0-0,025 ; 0,025-0,05 ; 0,05-0,10$; e 0,10 $0,20 \mathrm{~m}$, tomando-se o cuidado de preservar a estrutura do solo, que foi seco ao ar, e, posteriormente, os agregados foram passados em peneira de $9,51 \mathrm{~mm}$ e submetidos ao fracionamento físico densimétrico e à análise de estabilidade de agregados.

Utilizou-se o método de fracionamento densimétrico da MO, descrito em Conceição et al. (2008), que consistiu de agitação manual leve do tubo de centrífuga contendo $10 \mathrm{~g}$ de solo e $80 \mathrm{~mL}$ de solução de politungstato de sódio (PTS) com densidade de $2,00 \mathrm{~g} \mathrm{~cm}^{-3}$. Após a centrifugação (2.000 g por $1 \mathrm{~h}$ ), o sobrenadante, contendo a fração leve livre (FLL), foi filtrado (filtro de fibra de vidro $0,45 \mu \mathrm{m}$, previamente pesado). A solução de PTS foi recolocada sobre o material que restou no tubo que foi submetido à dispersão por sonicação com energia de $250 \mathrm{~J} \mathrm{~mL}^{-1}$ (previamente determinada para obtenção da máxima dispersão de agregados desse solo). Após a dispersão, o material foi centrifugado e filtrado novamente para a obtenção da fração leve oclusa (FLO). A fração restante no tubo de centrífuga correspondeu à fração pesada (FP). As FLL e FLO foram secas em estufa a $50{ }^{\circ} \mathrm{C}$ e moídas em gral. Os teores de carbono orgânico total (COT) do solo e das frações FLL e FLO foram determinados por combustão seca, em analisador elementar da marca Leco. O teor de C da FP foi obtido por diferença entre o COT e as frações leves [FP = (COT-(FLL+FLO)].

Os estoques de COT e nas frações físicas da MO foram calculados em massa equivalente de solo, segundo Sisti et al. (2004), utilizando-se a densidade do solo em cada camada e tratamento (Quadro 1), tendo o solo sob vegetação natural (SN) como referência. 
Quadro 1. Densidade do solo e diâmetro médio ponderado de agregados (DMP) nas quatro camadas dos tratamentos amostrados de um Planossolo Háplico sob diferentes sistemas de manejo

\begin{tabular}{|c|c|c|c|c|c|c|c|c|}
\hline \multirow{2}{*}{ Profundidade } & \multicolumn{4}{|c|}{ Densidade do solo ${ }^{(1)}$} & \multicolumn{4}{|c|}{ DMP } \\
\hline & ST & APC & APD & SN & ST & APC & APD & SN \\
\hline $\mathrm{m}$ & \multicolumn{4}{|c|}{$-\mathrm{Mg} \mathrm{m}^{-3}$} & \multicolumn{4}{|c|}{$-\mathrm{mm}$} \\
\hline $0-0,025$ & 1,55 & 1,49 & 1,51 & 1,49 & 2,44 & 2,01 & 2,56 & 3,89 \\
\hline $0,025-0,05$ & 1,50 & 1,45 & 1,48 & 1,54 & 2,52 & 1,33 & 3,80 & 3,65 \\
\hline $0,05-0,10$ & 1,53 & 1,53 & 1,52 & 1,55 & 2,50 & 2,21 & 1,16 & 2,33 \\
\hline $0,10-0,20$ & 1,57 & 1,60 & 1,54 & 1,57 & 2,17 & 1,88 & 1,15 & 1,95 \\
\hline
\end{tabular}

ST: Sistema tradicional de cultivo: um ano com arroz irrigado por alagamento (preparo convencional), seguido de dois anos de pousio; APC: Sistema de cultivo contínuo de arroz irrigado por alagamento (preparo convencional); APD: Sucessão de azevém x arroz irrigado, sob plantio direto; e SN: Solo sob vegetação nativa, sem cultivo. ${ }^{(1)}$ Fonte: Bamberg et al. (2009).

Foi estimada a participação relativa do estoque de $\mathrm{C}$ em cada fração em relação ao estoque de COT [(estoque fração/estoque COT) x 100].

A estabilidade de agregados em água foi determinada seguindo-se o método descrito por Kemper \& Rosenau (1986) modificado por Palmeira et al. (1999), o qual utiliza o aparelho de oscilação vertical de Yoder (1936), sendo neste trabalho representada pelo diâmetro médio ponderado (DMP) (Quadro 1).

Os resultados foram submetidos à análise de variância e quando significativos, aplicados o teste de Duncan a $5 \%$, para avaliação das diferenças entre os resultados obtidos nos tratamentos. Também foi realizada a análise de correlação entre os teores de COT e nas frações da MO, com o DMP, para observar se havia relação entre os mecanismos de estabilidade da MO e a agregação nos solos de várzea.

\section{RESULTADOS E DISCUSSÃO}

Os estoques de COT nas camadas $(0-0,025$ e 0,025$0,05 \mathrm{~m}$ ) foram influenciados pelo sistema de cultivo (Quadro 2). No sistema APD, esses valores foram superiores aos obtidos nos sistemas sob preparo convencional (ST e APC). Nas camadas de 0,05-0,10 e 0,10-0,20 m não houve diferença significativa entre os sistemas de cultivo.

Somando os estoques de COT nas diferentes profundidades em cada tratamento (Quadro 2), temse o estoque de COT na camada de $0-0,20 \mathrm{~m}$, que no sistema SN foi de $47,8 \mathrm{Mg} \mathrm{ha}^{-1}$. Esses valores pouco diferiram dos obtidos por outros autores nessa mesma camada. Zschornack (2007), avaliando solos de várzea sob campo nativo, obteve estoques de 47,34 e 64,34 Mg ha-1 em dois Planossolos distintos, localizados nos municípios de Pelotas e Cristal, de climas semelhantes ao do local deste trabalho. Conceição (2006) obteve um estoque de 39,32 $\mathrm{Mg} \mathrm{ha}^{-1} \mathrm{em}$ Argissolo, sob campo nativo, no município de Eldorado do Sul. Em três Latossolos distintos sob vegetação
Quadro 2. Estoque de carbono orgânico total (COT) nas frações leve livre (FLL) e leve oclusa (FLO) e na fração pesada (FP) da matéria orgânica nas camadas amostradas dos diferentes sistemas de cultivo de um Planossolo Háplico. Capão do Leão, RS

\begin{tabular}{|c|c|c|c|c|}
\hline $\begin{array}{l}\text { Sistema de } \\
\text { cultivo }\end{array}$ & $\operatorname{CoT}^{(1)}$ & FLL $^{(1)}$ & FLO $^{(1)}$ & $\mathrm{FP}^{(1)}$ \\
\hline & \multicolumn{4}{|c|}{$-\mathrm{Mg} \mathrm{ha}^{-1}$} \\
\hline & \multicolumn{4}{|c|}{$0-0,025 \mathrm{~m}$} \\
\hline $\mathrm{ST}$ & $5,58 \mathrm{c}$ & $0,52 \mathrm{c}$ & $1,09 \mathrm{a}$ & $3,97 \mathrm{~b}$ \\
\hline APC & $5,80 \mathrm{c}$ & $0,45 \mathrm{c}$ & $1,15 \mathrm{a}$ & $4,20 \mathrm{~b}$ \\
\hline APD & $11,11 \mathrm{~b}$ & $3,25 \mathrm{a}$ & $2,01 \mathrm{a}$ & $5,85 \mathrm{~b}$ \\
\hline SN & $17,73 \mathrm{a}$ & $1,59 \mathrm{~b}$ & $1,14 \mathrm{a}$ & $15,00 \mathrm{a}$ \\
\hline & \multicolumn{4}{|c|}{$0,025-0,05 \mathrm{~m}$} \\
\hline $\mathrm{ST}$ & $4,71 \mathrm{~b}$ & $0,35 \mathrm{~b}$ & $0,86 \mathrm{ab}$ & $3,50 \mathrm{~b}$ \\
\hline $\mathrm{APC}$ & $4,37 \mathrm{~b}$ & $0,30 \mathrm{~b}$ & $0,69 \mathrm{~b}$ & $3,38 \mathrm{~b}$ \\
\hline APD & $8,69 \mathrm{a}$ & $0,83 \mathrm{a}$ & $1,09 a b$ & $6,77 \mathrm{a}$ \\
\hline $\mathrm{SN}$ & $7,57 \mathrm{a}$ & $0,84 \mathrm{a}$ & $1,16 \mathrm{a}$ & $5,57 \mathrm{a}$ \\
\hline & \multicolumn{4}{|c|}{$0,05-0,10 \mathrm{~m}$} \\
\hline ST & $8,48 \mathrm{a}$ & $0,61 \mathrm{a}$ & $1,42 \mathrm{a}$ & $6,45 \mathrm{a}$ \\
\hline APC & $8,34 \mathrm{a}$ & $0,80 \mathrm{a}$ & $1,58 \mathrm{a}$ & $5,96 \mathrm{a}$ \\
\hline APD & $9,95 \mathrm{a}$ & $0,92 \mathrm{a}$ & $1,59 \mathrm{a}$ & $7,44 \mathrm{a}$ \\
\hline SN & $9,58 \mathrm{a}$ & $0,53 \mathrm{a}$ & $1,52 \mathrm{a}$ & $7,53 \mathrm{a}$ \\
\hline & \multicolumn{4}{|c|}{$0,10-0,20 \mathrm{~m}$} \\
\hline $\mathrm{ST}$ & $13,02 \mathrm{a}$ & $0,58 a b$ & $2,68 \mathrm{a}$ & $9,56 \mathrm{a}$ \\
\hline APC & $15,25 \mathrm{a}$ & $0,91 \mathrm{a}$ & $2,61 \mathrm{a}$ & $11,73 \mathrm{a}$ \\
\hline APD & $13,52 \mathrm{a}$ & $0,39 \mathrm{~b}$ & $2,16 \mathrm{a}$ & $10,97 \mathrm{a}$ \\
\hline $\mathrm{SN}$ & $12,95 \mathrm{a}$ & $0,68 \mathrm{ab}$ & $2,48 \mathrm{a}$ & $9,79 \mathrm{a}$ \\
\hline & \multicolumn{4}{|c|}{$0-0,20 \mathrm{~m}^{(2)}$} \\
\hline ST & $31,79 \mathrm{~b}$ & $2,06 \mathrm{c}$ & $6,06 \mathrm{a}$ & $23,68 \mathrm{c}$ \\
\hline APC & $33,75 \mathrm{~b}$ & $2,46 \mathrm{c}$ & $6,03 \mathrm{a}$ & $25,27 \mathrm{bc}$ \\
\hline APD & $43,27 \mathrm{a}$ & $5,39 \mathrm{a}$ & $6,85 \mathrm{a}$ & $31,03 \mathrm{~b}$ \\
\hline $\mathrm{SN}$ & $47,83 \mathrm{a}$ & $3,64 \mathrm{~b}$ & $6,30 \mathrm{a}$ & $37,89 \mathrm{a}$ \\
\hline
\end{tabular}

(1) Valores corrigidos pela massa equivalente de solo em cada camada (Sisti et al., 2004). ${ }^{(2)}$ Valores obtidos pela soma das quatro camadas amostradas. Médias seguidas da mesma letra na coluna, em cada camada, não diferem estatisticamente pelo teste de Duncan a $5 \%$. ST: Sistema tradicional de cultivo: um ano com arroz irrigado por alagamento (preparo convencional), seguido de dois anos de pousio; APC: Sistema de cultivo contínuo de arroz irrigado por alagamento (preparo convencional); APD: Sucessão de azevém x arroz irrigado, sob plantio direto; e SN: Solo sob vegetação nativa, sem cultivo. 
nativa, em municípios do Mato Grosso do Sul, Boeni (2007) verificou estoques de 44,56; 66,34; e $53,12 \mathrm{Mg}_{\text {ha }}$. Cabe salientar que a comparação com o Argissolo e o Latossolo deve ser vista com cautela, tendo em vista as diferenças inerentes de cada tipo de solo e clima local.

No sistema de referência ( $\mathrm{SN})$ e no $\mathrm{APD}$, o estoque de COT somado das camadas de 0-0,025 e 0,0250,05 m representou, respectivamente, 53 e $46 \%$ do $\mathrm{C}$ estocado na camada de $0-0,20 \mathrm{~m}$, enquanto nos sistemas com preparo convencional do solo (ST e APC) o estoque de COT somado das duas camadas superficiais foi de $32 \%$ em relação ao total estocado na camada arável. A manutenção da cobertura vegetal e o não revolvimento do solo são responsáveis por maior incremento de COT no solo e por menores taxas de decomposição, favorecendo, assim, a manutenção/recuperação dos teores de MO.

$\mathrm{Na}$ camada superficial (0-0,025 m), o estoque de C na FLL no sistema APD foi o dobro do SN, já os sistemas com preparo convencional, ST e APC, apresentaram estoques de $\mathrm{C}$ nessa fração bastante inferiores quando comparados aos SN (Quadro 2). Tal resultado evidencia que em solos de várzea cultivados com arroz irrigado, apesar de o alagamento reduzir a velocidade de decomposição dos resíduos orgânicos, o revolvimento do solo durante o preparo promove perdas consideráveis de carbono na camada superficial, principalmente da fração mais lábil (FLL). Entretanto, o acúmulo na superfície da palha do azevém cultivado no inverno no sistema APD contribuiu para o aumento do estoque de C da FLL. Devido à sua relativa facilidade de decomposição, o conteúdo de carbono nessa fração é dependente, em termos de dinâmica, do suprimento de resíduos orgânicos do sistema (Christensen, 2000).

Não houve diferença significativa, na camada de 0-0,025 m, entre os sistemas de manejo dos estoques de $\mathrm{C}$ da FLO. No entanto, o estoque de $\mathrm{C}$ da FP no $\mathrm{SN}$ foi três vezes maior do que a média dos estoques de $\mathrm{C}$ dos demais sistemas, indicando que nestes houve perda de C dessa fração. A estabilidade do SN sem utilização agrícola deve ter contribuído para a manutenção da MO nessa fração, uma vez que o solo não foi revolvido nesses 21 anos, mantendo a matéria orgânica protegida nos agregados e aumentando o período de interação do material particulado com os argilominerais - predomínio de caulinita e mistura de minerais $2: 1$, esmectita com polímeros entre camadas (Castilhos et al., 2002). No solo não cultivado, devido ao maior tempo de ciclagem da MOS nos macroagregados as reações de complexação entre as frações mineral e orgânica ocorreram mais efetivamente (Six et al., 1998).

Na camada de 0,025-0,05 m, os estoques das FLL e FP foram superiores nos sistemas SN e APD. Na fração FLO, essa tendência também foi observada, apesar de ter havido diferença significativa apenas entre os sistemas SN e APC. Em geral, os estoques da FLO foram superiores aos da FLL, o que pode ser consequência da maior eficiência do mecanismo de oclusão para a estabilização da MO nessa fração.

Na camada de 0,05-0,10 m não houve influência dos sistemas de manejo sobre os estoques de carbono nas frações densimétricas da MO. Na camada de 0,10 0,20 m, o estoque da FLL foi maior no APC em relação ao no APD, os quais não diferiram dos demais sistemas. O revolvimento do solo por ocasião do preparo convencional permitiu a incorporação do resíduo que estava na superfície, favorecendo o incremento dessa fração em profundidade.

Independentemente da profundidade avaliada, com exceção do APD e SN na camada de 0-0,025 m, os estoques de $\mathrm{C}$ na FLL, FLO e FP obedeceram à seguinte ordem: FP $>$ FLO $>$ FLL. Essa sequência estaria de acordo com os diferentes graus de estabilidade da MO em cada fração (Roscoe et al., 2006). Embora a metodologia adotada neste trabalho não seja completamente eficiente para avaliar a estabilidade da matéria orgânica do solo, é provável que na FLL apenas a recalcitrância química seja responsável pela estabilidade da MO, enquanto na FLO a proteção física, ou oclusão da MO no interior dos agregados do solo, também estaria contribuindo para a manutenção da MOS. Já na fração pesada os três mecanismos de estabilização - recalcitrância, oclusão e interação da MO com os minerais do solo - poderiam atuar conjuntamente (Nascimento et al., 2009). Em decorrência disso, a FP, seguida da FLO, possuiria um tempo maior de ciclagem do que a FLL. Resultados semelhantes foram observados por Conceição et al. (2008) em Argissolo Vermelho e Latossolo Vermelho submetidos aos sistemas de cultivo convencional e plantio direto. $\mathrm{O}$ fato de os sistemas APD e SN terem apresentado maior estoque da FLL em relação à FLO é explicado pelo grande aporte de resíduo na superfície do solo, associado a um ambiente maldrenado, em que a alta umidade do solo de várzea e o não revolvimento contribuem para uma taxa de decomposição mais lenta.

A proporção dos estoques de $\mathrm{C}$ em cada fração em relação ao COT encontra-se na figura 1. A FLL representou a menor proporção do COT entre os sistemas (9\% em média), em todas as profundidades e tratamentos, exceto no APD da camada superficial. A FLO, apesar de ter sido mais expressiva do que a FLL na maioria dos sistemas e profundidades, em média representou $17 \%$ do COT. Já a FP, na média dos tratamentos, representou $74 \%$ do estoque de COT, sendo a mais importante para a manutenção dos estoques de carbono nesse solo.

Esses resultados diferem dos de Nascimento et al. (2009), que encontraram proporções similares entre FLL e FLO (17 e 18 \%) em um Gleissolo com cultivo de arroz irrigado, no município de Cachoeirinha, RS. Esses autores concluíram que a proteção física foi pouco expressiva para a manutenção da MOS. Já em Latossolos Boeni (2007) observou proporções 
significativamente maiores da FLO em relação à FLL (em média 19,5 e 5,4\%, respectivamente).

Os teores de COT e C das frações físicas da MO não se correlacionaram com o diâmetro médio ponderado do solo (DMP) (Quadro 1) nos sistemas de cultivo de arroz com preparo convencional (ST e APC) (Figura 2), mostrando que, possivelmente, a MO não está contribuindo para a estabilização dos agregados do solo e, ou, a agregação do solo nesses sistemas. O intenso revolvimento do solo provocou a quebra dos agregados, expondo a MO, o que diminuiu a proteção física e o tempo de contato da fração orgânica com a mineral para que houvesse interação entre elas.

No sistema em que o solo não é cultivado (SN), observou-se, entretanto, correlação entre o DMP e o COT, FLL e FP (Figura 2). Esse resultado, comparado ao encontrado nos sistemas com revolvimento do solo, evidencia a fragilidade desse solo quanto à sua eficiência na proteção da MO, em que a utilização intensiva no cultivo agrícola, além de causar perdas significativas nos estoques de $\mathrm{C}$ no solo, reduz a sua capacidade de proteger a MOS.

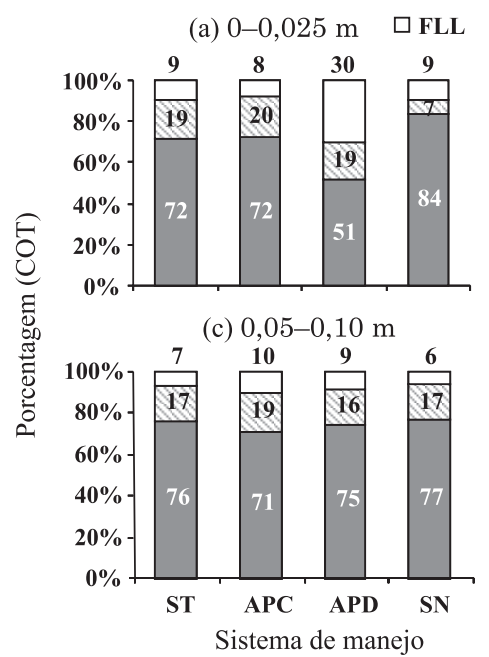

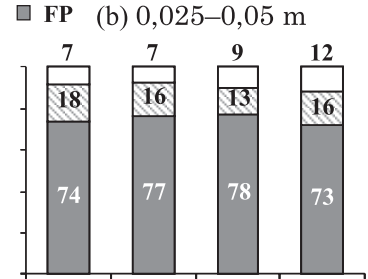

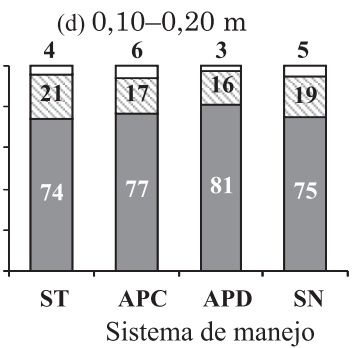

Figura 1. Proporção do carbono orgânico total (COT) nas frações leve livre (FLL), leve oclusa (FLO) e pesada (FP) da matéria orgânica de um Planossolo submetido a diferentes sistemas de cultivo. ST: Sistema tradicional de cultivo: um ano com arroz irrigado por alagamento (preparo convencional), seguido de dois anos de pousio; APC: Sistema de cultivo contínuo de arroz irrigado por alagamento (preparo convencional); APD: Sucessão de azevém x arroz irrigado por alagamento (plantio direto); e $\mathrm{SN}$ : Solo sob vegetação nativa (sem cultivo).
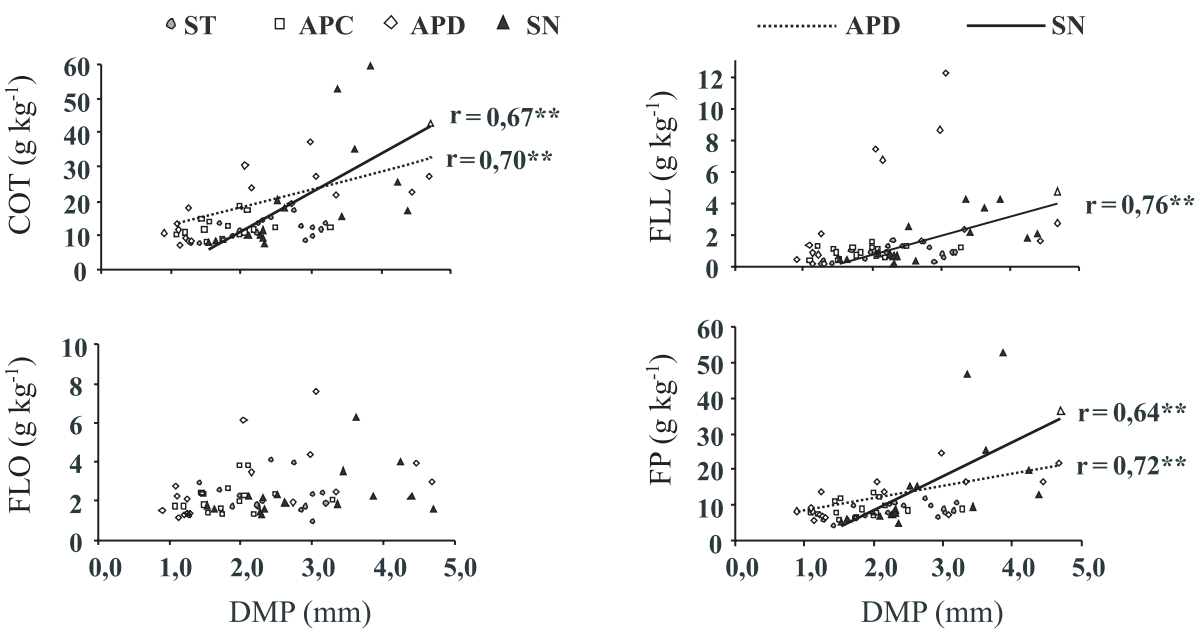

Figura 2. Correlação entre o diâmetro médio ponderado (DMP) dos agregados de um Planossolo sob diferentes sistemas de manejo e o teor de carbono orgânico total (COT) e das frações leve livre (FLL), leve oclusa (FLO) e pesada (FP) da matéria orgânica do solo. ** Significativo < 1 \%. ST: Sistema tradicional de cultivo: um ano de arroz irrigado por alagamento (preparo convencional), seguido de dois anos de pousio; APC: Sistema de cultivo contínuo de arroz irrigado por alagamento (preparo convencional); APD: Sucessão de azevém $x$ arroz irrigado por alagamento (plantio direto); e SN: Solo sob vegetação nativa (sem cultivo). 
No APD foram encontradas associações do DMP com o COT $\left(r=0,70^{* *}\right)$ e com a FP $\left(r=0,72^{* *}\right)$ (Figura 2), mostrando que cerca de $70 \%$ da variação do DMP se correlaciona positivamente com a variação do COT, o que pode indicar que boa parte da estabilidade dos agregados se deva à MO total no primeiro caso e à fração pesada no segundo. Martins et al. (2009) também encontraram correlações positivas entre o diâmetro médio ponderado de agregados e os teores de COT em um Latossolo, sob plantio direto.

A FLO não apresentou correlação com o DMP em nenhum dos sistemas de manejo estudados, sugerindo que, provavelmente, a oclusão seria um mecanismo de preservação da MO pouco eficiente nos solos de várzea, uma vez que a umidade excessiva e o alagamento por ocasião do cultivo do arroz proporcionam agregação transitória entre cultivos.

\section{CONCLUSÕES}

1. O sistema de manejo com a sucessão arroz irrigado e azevém em plantio direto após 21 anos é mais eficiente na preservação dos estoques de COT e da fração leve livre, nas camadas do solo até $0,05 \mathrm{~m}$, em comparação com os sistemas de arroz irrigado com preparo convencional.

2. No Planossolo estudado, os sistemas de cultivo de arroz irrigado não afetaram os estoques de $\mathrm{C}$ da fração leve oclusa.

3. A quantidade de CO no Planossolo, em todos os sistemas de cultivo e nas profundidades avaliadas, deve-se, principalmente, à fração pesada.

\section{AGRADECIMENTOS}

À Fundação de Amparo à Pesquisa do Estado do Rio Grande do Sul, ao Conselho Nacional de Desenvolvimento Científico e Tecnológico (CNPq) e ao Ministério da Ciência e Tecnologia (MCT), pelo auxílio financeiro no âmbito do projeto PRONEX 04/850.0 "Sequestro de carbono e mitigação das emissões de gases de efeito-estufa por sistemas conservacionistas de manejo e as oportunidades para o agronegócio no Rio Grande do Sul" -; e à CAPES e ao CNPq, pela concessão das bolsas de doutorado e mestrado.

\section{LITERATURA CITADA}

BAMBERG, A.L.; PAULETO, E.A.; GOMES, A. S.G.; TIMM, L.C.; PINTO, L.F.S.; LIMA, A.C.R. \& SILVA, T.R. Densidade de um Planossolo sob sistemas de cultivo avaliada por meio da tomografia computadorizada de raios gama. R. Bras. Ci. Solo, 33:1079-1086, 2009.
BOENI, M. Proteção física da matéria orgânica em Latossolos sob sistemas com pastagens na região do cerrado brasileiro. Porto Alegre, Universidade Federal do Rio Grande do Sul, 2007. 136p. (Tese de Doutorado)

CASTILHOS, R.M.V.; MEURER, E.J.; KÄMPF, N. \& PINTO, L.F.S. Mineralogia e fontes de potássio em solos no Rio Grande do Sul cultivados com arroz irrigado. R. Bras. Ci. Solo, 26:579-587, 2002.

CHRISTENSEN, B.T. Organic matter in soil: Structure, function and turnover. Tjele, Danish Institute of Agricultural Sciences Denmark, 2000. 95p. (Report Plant Production, 30)

CONCEIÇÃO, P.C. Agregação e proteção física da matéria orgânica em dois solos do sul do Brasil. Porto Alegre, Universidade Federal do Rio Grande do Sul, 2006. 138p. (Tese de Doutorado)

CONCEIÇÃO, P.C.; BOENI, M.; DIECKOW, J.; BAYER, C. \& MIELNICZUK, J. Fracionamento densimétrico com politungstato de sódio no estudo da proteção física da matéria orgânica em solos. R. Bras. Ci. Solo, 32:541-549, 2008.

GAUNT, J.L.; NEUE, H.U. \& BRAGAIS, J. Soil characteristics that regulate soil reduction and methane production in wetland rice soils. Soil Sci. Soc. Am. J., 61:1526-1531, 1997.

KEMPER, W.D. \& ROSENAU, R.C. Aggregate stability and size distribution. In: KLÜTE, A., ed. Methods of soil analysis. 2.ed. Madison, Soil Science Society of America, 1986. p.425-441.

MARTINS, M.R.; CORÁ, J.E.; JORGE, R.F. \& MARCELO, A.V. Crop type influences soil aggregation and organic matter under no-tillage. Soil Tillage Res., 104:22-29, 2009.

NASCIMENTO, P.C.; BAYER, C.; NETTO, L.F.S.; VIAN, A.C.; VIEIRO, F.; MACEDO, V.C.M. \& MARCOLIN, E. Sistemas de manejo e a matéria orgânica de solo de várzea com cultivo de arroz. R. Bras. Ci. Solo, 33:1821-1827, 2009.

PALMEIRA, P.R.T.; PAULETTO, E.A.; TEIXEIRA, C.F.A.; GOMES, A.S. \& SILVA, J.B. Agregação de um Planossolo submetido a diferentes sistemas de cultivo. R. Bras. Ci. Solo, 23:189-195, 1999.

PINTO, L.F.S.; LAUS NETO, J.A. \& PAULETTO, E.A. Solos de várzea do Sul do Brasil cultivados com arroz irrigado. In: GOMES, A.S. \& MAGALHÃES JUNIOR, A.M., ed. Arroz irrigado no Sul do Brasil. Pelotas, Embrapa Clima Temperado, 2004. p.75-95.

ROSCOE, R.; MADARI, B.E. \& MACHADO, P.L.O.A. Fracionamento físico do solo na obtenção de compartimentos mensuráveis para uso em simuladores da dinâmica da matéria orgânica. In: ROSCOE, R.; MERCANTE, F.M. \& SALTON, J.C., ed. Dinâmica da matéria orgânica do solo em sistemas conservacionistas: Modelagem matemática e métodos auxiliares. Dourados, Embrapa Agropecuária Oeste, 2006. p.107-132.

SISTI, C.P.J.; SANTOS, H.P.; KOHHANN, R.; ALVES, B.J.R.; URQUIAGA, S. \& BODDEY, R.M. Change in carbon and nitrogen stocks in soil under 13 years of conventional or zero tillage in southern Brazil. Soil Tillage Res., 76:39-58, 2004. 
SIX, J.; ELLIOTT, E.T.; PAUSTIAN, K. \& DORAN, J.W. Aggregation and soil organic matter accumulation in cultivaded and native grassland soils. Soil Sci. Soc. Am. J., 62:1367-1377, 1998.

TISDALL, J.M. \& OADES, J.M. Organic matter and waterstable aggregates in sols. J. Soil. Sci., 33:141-163, 1982.
YODER, R.E. A direct method of aggregate analysis of soils and a study of the physical nature of erosion losses. J. Am. Soc. Agron., 28:337-351, 1936.

ZSCHORNACK, T. Fracionamento e estoques de carbono orgânico de solos de várzea sob campo natural no Rio Grande do Sul. Pelotas, Universidade Federal de Pelotas, 2007. 88p. (Tese de Mestrado) 